\title{
Skin denervation, neuropathology, and neuropathic pain in a laser-induced focal neuropathy
}

\author{
Hou-Yu Chiang, ${ }^{\mathrm{a}}$ Chin-Tin Chen, ${ }^{\mathrm{b}}$ Hsiung-Fei Chien, ${ }^{\mathrm{a}, \mathrm{c}}$ and Sung-Tsang Hsieh ${ }^{\mathrm{a}, \mathrm{d}, *}$ \\ ${ }^{a}$ Department of Anatomy and Cell Biology, National Taiwan University College of Medicine, Taipei 10018, Taiwan \\ ${ }^{\mathrm{b}}$ Center for Optoelectronic Biomedicine, National Taiwan University College of Medicine, Taipei 10018, Taiwan \\ ${ }^{c}$ Department of Surgery, National Taiwan University Hospital, Taipei 10002, Taiwan \\ ${ }^{\mathrm{d}}$ Department of Neurology, National Taiwan University Hospital, Taipei 10002, Taiwan
}

Received 21 April 2004; revised 23 July 2004; accepted 13 September 2004

Available online 19 November 2004

\begin{abstract}
Small-diameter sensory nerves innervating the skin are responsive to noxious stimuli, and an injury to these nerves is presumably related to neuropathic pain. Injury-induced neuropathic pain in animals can be produced by laser irradiation, which usually requires concomitant use of photosensitive dyes, known as the photochemical approach. It is not clear whether laser irradiation alone can induce neuropathic pain. In addition, two issues are important to apply these approaches: the relationship between the extent of laser irradiation and the occurrence of neuropathic pain, and the susceptibility of small-diameter sensory nerves in the skin to laser-induced neuropathic pain. To address these issues, we designed a new model of focal neuropathy by applying a diode laser of $532 \mathrm{~nm}(100 \mathrm{~mW})$ to the sciatic nerve and evaluated small-diameter nerves by quantifying skin innervation and largediameter nerves by measuring amplitudes of the compound muscle action potential (CMAP). Immediately after laser irradiation, epineurial vessels were occluded due to the formation of thrombi, and the blood flow through these vessels was markedly reduced. On postoperative day (POD) 2, animals developed characteristic manifestations of neuropathic pain, including spontaneous pain behaviors, thermal hyperalgesia, and mechanical allodynia. These phenomena peaked during PODs 7-21, and lasted for 3-6 weeks. The neuropathology at the irradiated site of the sciatic nerve included a focal area of axonal degeneration surrounded by demyelination and endoneurial edema. The extent of damage to large-diameter motor and sensory nerves after laser irradiation was evaluated by nerve conduction studies. On the irradiated sides, amplitudes of the compound muscle action potentials and sensory nerve action potentials (SNAPs) were reduced to $65.0 \%(P<0.0001)$ and $42.5 \%(P<0.01)$ of those on the control sides, respectively. Motor innervation of the neuromuscular junctions (NMJs) on plantar muscles was examined by combined cholinesterase histochemistry and immunohistochemistry. The ratio of innervated NMJs on the operated sides decreased to $76.3 \%$ of that on
\end{abstract}

* Corresponding author. Department of Anatomy and Cell Biology, National Taiwan University College of Medicine, 1 Jen-Ai Road, Sec. 1, Taipei 10018, Taiwan. Fax: +886223915292.

E-mail address: sthsieh@ntumc.org (S.-T. Hsieh).

Available online on ScienceDirect (www.sciencedirect.com). the control side. Skin innervation in the territory of the irradiated sciatic nerves was evaluated by immunohistochemistry with neuronal markers. Among these markers, epidermal nerve densities for protein gene product (PGP) 9.5, calcitonin gene-related peptide (CGRP), and substance $P$ (SP) were significantly lower on the irradiated sides than the control sides with a different degree of loss for each marker (42.1$\mathbf{5 3 . 1 \%}, P<\mathbf{0 . 0 5}$ ). Results suggest that laser-induced focal neuropathy provides a new system for studying neuropathic pain. With this approach, the extent of nerve injury can be quantified. Both smalldiameter epidermal nerves and large-diameter sensory and motor nerves are susceptible to laser-induced injury of different degrees.

(c) 2004 Elsevier Inc. All rights reserved.

Keywords: Skin innervation; Epidermal nerves; Laser irradiation; Neuropathic pain; Ischemic neuropathy; Ubiquitin; Protein gene product 9.5

\section{Introduction}

Small-diameter sensory nerves of the skin are responsible for conveying noxious and thermal stimuli. Damage to these nerves is presumably related to neuropathic pain behaviors. An understanding of pain mechanisms requires experimental systems to assess the relationship between nerve injury and neuropathic pain and to explore new therapeutic strategies. A critical issue in neuropathic pain studies is to develop an experimental system which produces a quantifiable degree of injury.

Laser irradiation together with photosensitive dyes has become a new approach to induce tissue injury for clinical use, which is known as photodynamic therapy (Dolmans et al., 2003). Only a few reports have employed this approach to induce painful neuropathies (Gazelius et al., 1996; Hao et al., 2000; Kupers et al., 1998). One potential issue is the concomitant requirement of photosensitive dyes. Rosen et al. (2001) showed that laser irradiation alone could elicit damage to endothelial cells of capillaries and small arteries resulting in thrombosis. Previous 
studies employing laser irradiation indicated that $\mathrm{CO}_{2}$ laser alone could induce Wallerian degeneration of varying degrees (Menovsky et al., 1996, 2000; Myers et al., 1985). Those studies did not specify whether neuropathic pain developed in animals after laserinduced nerve injury but raised the possibility that laser irradiation alone under appropriate conditions may produce a new experimental system of inducing neuropathic pain.

Neuropathic pain results in a series of complex but coordinated behaviors mediated by damage to large myelinated, small myelinated, and unmyelinated nerve fibers (Woolf, 2000). We and others have demonstrated the rich innervation of the skin by immunohistochemistry with various neuronal markers, particularly, protein gene product (PGP) 9.5 (Hilliges et al., 1995; Hsieh et al., 2000; Kennedy and Said, 1999; Vaalasti et al., 1988; Wang et al., 1990). PGP 9.5 is a ubiquitin carboxyhydrolase and probably functions as an immediate early gene for processing sensory information in the neurons (Hegde et al., 1997). Our previous study on chronic constriction injury indicates that PGP 9.5(+) nerve terminals in the skin were moderately depleted compared with those in completely denervated skin (Lin et al., 2001). These findings suggest that partial injury is another important principle for creating experimental models of neuropathic pain. An open issue is whether sensory nerve terminals in the skin of different phenotypes, such as nerves positive for calcitonin gene-related peptide (CGRP) and substance P (SP), are depleted to the same degree in neuropathic pain. Apparently, large-diameter and smalldiameter nerve fibers are differentially vulnerable in models of neuropathic pain (Basbaum et al., 1991; Gautron et al., 1990; Guilbaud et al., 1993; Nuytten et al., 1992). Whether terminals of sensory nerves in the skin retain the same patterns as they have at the sciatic nerve level is an open issue. Thus, it was intriguing to investigate whether there is a relationship between nerve injury to fibers of different categories and the magnitude of neuropathic pain.

To address the above issues, we developed a new system of neuropathic pain by laser irradiation alone and took multidisciplinary approaches to evaluate small-diameter sensory nerves by examining the innervation of the skin and large-diameter motor nerves by examining the innervation of motor endplates.

\section{Materials and methods}

\section{Animals}

The experiments were performed on adult male SpragueDawley rats weighing 200-250 g. Animals were housed in plastic cages whose floors were covered with sawdust to avoid mechanical damage to the hind paw skin. Sufficient water and food were provided. The experiments followed the guidelines of the International Association for the Study of Pain (IASP) (IASP Committee, 1980; Zimmermann, 1983).

\section{Laser-induced sciatic nerve injury}

Rats were anesthetized by an intraperitoneal injection of chloral hydrate $(400 \mathrm{mg} / \mathrm{kg})$. The sciatic nerve was exposed at the midthigh level after a dorsolateral skin incision and splitting of the fascia between the gluteus and biceps femoris muscle. The nerve was gently dissected from the surrounding connective tissues over a distance from the gluteus muscle to the trifurcation of the sciatic nerve. The segment of the sciatic nerve just distal to the gluteus muscle was marked with epineurial sutures and irradiated for various periods under a laser beam. The source of laser irradiations came from a diode-pumped solid state laser operating at $532 \mathrm{~nm}$ (HCP Corp., Hsinchu, Taiwan) with an output power of $100 \mathrm{~mW}$. The beam of the laser (1-mm diameter) was focused on the epineurial vessels of the sciatic nerve. For each animal, the operation was performed on one side, with the other side undergoing a sham operation. The intensity of the laser output was measured with a laser power meter (LaserCheck, 33-1553, Coherent, Auburn, CA) before and after the surgery. Sham operation was performed on control sides with similar procedures of removing connective tissues except that laser irradiation was omitted.

\section{Blood flow in sciatic nerves}

Epineurial blood flow of sciatic nerves was measured with a laser Doppler flowmeter (CAM1, KK Research Technology, Devon, England) before and immediately after laser irradiation (Morris et al., 1996). The sciatic nerve was exposed at the thigh level and covered with a pool of paraffin oil to prevent dryness of the nerve. A laser Doppler probe with a spot of $10 \mu \mathrm{m}$ in diameter was positioned perpendicularly to the nerve segment covered by a drop (approximately $50 \mu \mathrm{l}$ ) of liquid paraffin oil. This minimal amount of paraffin oil only covered the surface of the sciatic 7 nerve and did not interfere with the measurement of blood flow. During the 10-min examining period, no more paraffin oil was added. Before and after laser irradiation, blood flow data were collected for an interval of $2 \mathrm{~min}$, respectively. The surface of the sciatic nerves was still wet after the test.

\section{Behavioral testing}

Two measures were employed to assess thermal hyperalgesia and mechanical allodynia after irradiation (Chaplan et al., 1994; Hargreaves et al., 1988). Rats were adapted to the test environment for 5-7 days before testing. The baseline responses were recorded before irradiation of the sciatic nerve. After surgery, rats were tested on days 2, 4, and 7 and then weekly during the experimental period.

\section{Thermal hyperalgesia}

To evaluate the response to thermal stimulation, rats were assessed with the paw-withdrawal test of the Hargreaves type (Ugo Basile, Comerio, Italy) (Hargreaves et al., 1988; Lin et al., 1997). Animals were placed in a plastic box on a glass plate. The plantar surface of the hind paw was directly stimulated with an infrared source through the glass plate. Two parameters were evaluated: (1) the paw-withdrawal latency, defined as the interval between the onset of heat stimulation and withdrawal of the hind paw, and (2) the hind paw elevation time, defined as the interval between withdrawal of the hind paw and replacement of the paw on the floor. Both parameters were measured to the nearest $0.1 \mathrm{~s}$. The hind paws were tested in a random fashion. Each paw was tested five times with a 5-min interval between consecutive trials. The five withdrawal latencies per side were averaged. The difference between the two sides (the operated side minus the control side) was the withdrawal latency difference, with a value of $\geq 2 \mathrm{~s}$ defined as hyperalgesia. 
During the five tests, the intensity of the withdrawal responses was further categorized as 1 point for withdrawal with a brief pawlift, 2 points for a short-interval withdrawal $(\leq 5 \mathrm{~s})$ sometimes with a transient paw-lick, and 3 points for prolonged withdrawal ( $>5 \mathrm{~s})$ with vocalization, escaping, and prolonged licking of the tested paw, and sometimes with gentle biting. The weighted mean of the five responses was defined as the behavioral score.

\section{Mechanical allodynia}

To examine the response to mechanical stimulation, a set of 17 calibrated von Frey hairs $(0.026-110 \mathrm{~g}$, Somedic, Sweden) was used for assessment (Chaplan et al., 1994). Animals were placed in a plastic box on a metal mesh floor with a habituation period of $10 \mathrm{~min}$. The testing was initiated with the hair weighing $3.30 \mathrm{~g}$, and each foot was examined in a consecutive fashion with a descending or ascending hair number according to the response. Five stimuli using the selected hair were applied at 5-s intervals. If there was no withdrawal response to the initially selected hair with these five stimuli, a stronger stimulus was applied. If the animal withdrew its hind paw in response to any of the five stimuli, the next weaker stimulus was chosen. The mechanical threshold was expressed as the minimal force $(g)$ initiating a withdrawal response. The results were expressed as the logarithm of the withdrawal ratio (operated side over control side). Negative data indicated a reduction in the mechanical threshold of the operated side compared to the control side.

\section{Electrophysiological studies}

The motor function of the sciatic nerve was assessed weekly after the operation (Ko et al., 1999). Rats were anesthetized before evaluation, and the compound muscle action potential (CMAP) was measured with an evoked response recorder (Neuropack II, MEB-5100, Nihon Kohden, Tokyo, Japan). The stimulating electrodes were inserted and placed at the sciatic notch to stimulate the sciatic nerve, and the recording electrodes were on the plantar muscles. Amplitudes of the CMAP on both sides were recorded for analysis. Sensory nerve action potential (SNAP) of the sural nerve was recorded orthodromically. The sural nerve was exposed and gently dissected free from the surrounding connective tissues in the popliteal fossa. The stimulating surface electrodes were placed at the lateral side of the foot dorsum, distal to the lateral malleolus. The needle recording electrodes were placed beside the sural nerve near the sciatic nerve trifurcation. Amplitudes of the SNAP on both sides were recorded for analysis.

\section{Light and electron microscopic studies of nerve pathology}

Animals were perfused intracardially with $4 \%$ paraformalde-

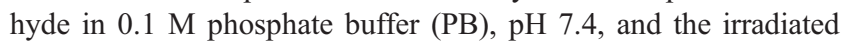
and distal parts of the sciatic nerve were postfixed in 5\% glutaraldehyde in $0.1 \mathrm{M}$ PB overnight (Lin et al., 1997). Tissue was postfixed in $2 \%$ osmic acid for $2 \mathrm{~h}$ at room temperature, dehydrated with a graded series of alcohol, and embedded in Epon 812 resin (Polyscience, Philadelphia, PA). Cross-sections of $1 \mu \mathrm{m}$ were cut on an ultramicrotome, dried on slides using a hot plate, stained with toluidine blue, and observed under a light microscope. Axonal degeneration, demyelination, and the degree of endoneurial edema were evaluated according to established criteria (Iida et al., 2003; Nakuda et al., 2002). Selected areas were thin-sectioned, doubly stained with uranyl acetate and lead citrate, observed under a Hitachi electron microscope, and photographed.

\section{Immunohistochemistry of footpads}

For immunohistochemistry on frozen microtome sections (Lin et al., 2001), animals were fixed with an intracardiac perfusion with $4 \%$ paraformaldehyde in $0.1 \mathrm{M}(\mathrm{PB}), \mathrm{pH} 7.4$. The skin areas innervated by the sciatic nerve were fixed for another $6 \mathrm{~h}$ and then changed to $\mathrm{PB}$ for storage. After thorough rinsing in $\mathrm{PB}$, samples were cryoprotected with $30 \%$ sucrose in PB overnight. Sections perpendicular to the epidermis were cut at $30 \mu \mathrm{m}$ on a sliding microtome, labeled sequentially, and stored at $-20^{\circ} \mathrm{C}$. To ensure adequate sampling, every fourth section for each tissue was chosen for immunohistochemistry. Sections were treated with $0.5 \%$ Triton $\mathrm{X}-100$ in $0.5 \mathrm{M}$ Tris buffer (Tris), $\mathrm{pH} 7.6$, for $30 \mathrm{~min}$ and processed for immunostaining. Briefly, sections were quenched with $1 \%$ $\mathrm{H}_{2} \mathrm{O}_{2}$ in methanol and blocked with $5 \%$ normal goat serum in $0.5 \%$ nonfat dry milk/Tris. Sections were incubated with rabbit antiserum to PGP 9.5 (UltraClone, Isle of Wight, UK, 1:1000), CGRP (Chemicon, Temecula, CA, 1:2000), SP (DiaSorin, Stillwater, MN, 1:1000), and growth-associated protein 43 (GAP 43) (Chemicon, Stillwater, MN, 1:1000) for 16-24 h. CGRP and SP are neuropeptides related to sensory perception (Ma and Bisby, 2000). GAP 43 is a neuronal presynaptic membrane protein that is generally considered to be a marker of neuronal plasticity (Doubell and Woolf, 1997). After rinsing in Tris, sections were incubated with biotinylated goat antirabbit $\operatorname{IgG}$ for $1 \mathrm{~h}$, and the avidin-biotin complex (Vector, Burlingame, CA) for another hour. The reaction product was demonstrated by 3,3-diaminobenzidine (DAB, Sigma, St. Louis, MO).

\section{Quantitation of epidermal innervation}

Epidermal innervation was quantified according to modified protocols in a coded fashion (Hsieh et al., 2000). PGP 9.5-, CGRP-, SP-, and GAP 43-immunoreactive nerves in the epidermis of each footpad were counted at a magnification of $\times 400$ with an Olympus BX40 microscope (Tokyo, Japan). Each individual nerve with branching points inside the epidermis was counted as one. For epidermal nerves with branching points in the dermis, each individual nerve was counted separately. The total length of the epidermis along the upper margin of the stratum corneum in each footpad was measured using the Image-Pro PLUS system (Media Cybernetics, Silver Spring, MD). Epidermal nerve density was therefore derived and expressed as the number of fibers per millimeter of epidermal length. Every fourth section of each tissue was quantified, and there were three sections for each footpad. All slides were coded during the quantitation.

\section{Combined cholinesterase histochemistry and immunohistochemistry}

For morphological examination of the motor innervation, cholinesterase histochemistry combined with immunohistochemistry was performed on the plantar muscles following established protocols (Ko et al., 1999). The plantar muscles were dissected after overnight postfixation. Serial $30-\mu \mathrm{m}$ cryostat sections were mounted on gelatin-coated slides. Every fifth section was stained with cholinesterase histochemistry and immunohistochemistry for PGP 9.5. This method demonstrates the neuromuscular junctions 
(NMJs) and motor innervation simultaneously. Coded sections from the control side and the operated side were observed at a magnification of $\times 400$ under an Olympus BX40 microscope. The ratios of innervated NMJs to total NMJs on each section were calculated for analysis.

\section{Experimental design and statistical analysis}

In the first phase of the experiment, we investigated the optimal duration of laser irradiation for inducing neuropathic pain. The criterion of successful induction was the presence of sustained thermal hyperalgesia at least in the interval between postoperative days (PODs) 7 and 14, when animals usually showed the maximal neuropathic pain behaviors according to previous studies on different neuropathic pain systems including chronic constriction injury (Bennett and Xie, 1988), partial sciatic nerve ligation (Seltzer et al., 1990), tight ligation of spinal nerves (Chaplan et al., 1994), and spared nerve injury (Decosterd and Woolf, 2000). Clearly, the success rate of inducing neuropathic pain depended on the duration of laser irradiation (Table 1). Irradiation with a power of $100 \mathrm{~mW}$ for $30 \mathrm{~s}$ induced the highest rate of neuropathic pain induction (four of six animals). Laser irradiation of a shorter duration (15 s) did not produce significant changes in the paw-withdrawal latencies on noxious heat stimulation. A longer duration of laser irradiation (60 s) generated various outcomes; three animals exhibited thermal hyperalgesia, and the others showed thermal anesthesia as reflected in the large variation in paw-withdrawal latencies. We therefore determined that laser irradiation of $100 \mathrm{~mW}$ for $30 \mathrm{~s}$ was the optimal dose and used this setting to induce neuropathic pain in the animals. In total, there were 62 animals (including the six rats in the firstphase experiment) subjected to laser irradiation of $100 \mathrm{~mW}$ for $30 \mathrm{~s}$, and 37 of them with an overall success rate of $59.7 \%$ fulfilled the criteria described above. These 37 animals were used for characterization of behavioral, electrophysiological, and pathological changes. At each time point, there were at least five animals for the laboratory procedures (semi-thin sections and immunohistochemistry of footpads). All procedures of measurement, quantitation, and analysis were performed in a blinded fashion. Behavioral and laboratory data were presented as the mean \pm SEM at different time points after laser irradiation. For statistical analysis of values

Table 1

Influence of laser irradiation duration for inducing neuropathic pain

\begin{tabular}{|c|c|c|c|}
\hline $\begin{array}{l}\text { Duration of laser } \\
\text { irradiation (s) }\end{array}$ & 15 & 30 & 60 \\
\hline \multicolumn{4}{|l|}{ Number of animals } \\
\hline Total & 5 & 6 & 9 \\
\hline $\begin{array}{l}\text { With thermal } \\
\text { hyperalgesia }^{a}\end{array}$ & 0 & 4 & 3 \\
\hline $\begin{array}{l}\text { Success rate for } \\
\text { inducing thermal } \\
\text { hyperalgesia }\end{array}$ & $0 \%$ & $66.7 \%$ & $33.3 \%$ \\
\hline \multicolumn{4}{|l|}{$\begin{array}{l}\text { Difference in } \\
\text { withdrawal } \\
\text { thresholds }(\mathrm{s})^{\mathrm{b}}\end{array}$} \\
\hline On POD $7^{\mathrm{b}}$ & $0.38 \pm 1.25$ & $-3.16 \pm 0.19$ & $1.64 \pm 2.7$ \\
\hline On POD $14^{b}$ & $1.80 \pm 1.13$ & $-2.79 \pm 0.24$ & $-0.65 \pm 1.68$ \\
\hline
\end{tabular}

\footnotetext{
a According to the difference in the withdrawal latency between the irradiated side and the control side on both postoperative days (POD) 7 and POD 14.

b Mean \pm SEM.
}

obtained from behavior testing over the experimental period, repeated-measures ANOVA followed by Dunn's post hoc test was used. Differences in the values in amplitude of CMAP and epidermal nerve density between control and operated sides were tested using Student $t$ test. Values of $P<0.05$ were considered significant.

\section{Results}

\section{Effect of laser irradiation on epineurial arteries and blood flow}

To understand pathophysiological consequences of irradiation with diode laser at $532 \mathrm{~nm}(100 \mathrm{~mW}, 30 \mathrm{~s})$ on epineurial vessels of sciatic nerves, we evaluated blood flow and vascular pathology. Before the application of laser, we measured the epineurial blood flow on both sides after removing connective tissues. The appearance of epineurial arteries was smooth (Figs. $1 \mathrm{~A}$ and $\mathrm{B})$. The blood flows through epineurial vessels on both sides were similar $(0.27 \pm 0.02 \mathrm{~mm} / \mathrm{s}$ on control side vs. $0.30 \pm$ $0.04 \mathrm{~mm} / \mathrm{s}$ on operated side, $P=0.53$; Fig. $1 \mathrm{D}, \mathrm{E})$. Three minutes after laser irradiation, the outline of epineurial vessels on the operated side became irregular, and the diameter was reduced (Fig. 1C). The blood flow through the irradiated segment of sciatic nerves was significantly reduced after laser irradiation compared with that before laser irradiation $(0.05 \pm 0.01 \mathrm{~mm} / \mathrm{s}$ vs. $0.30 \pm 0.04 \mathrm{~mm} / \mathrm{s}, P=0.0025$, Fig. 1E, F). These blood flow data were supported by ultrastructural evidence of thrombosis in epineurial vessels. Immediately after laser irradiation, thrombi were detected in epineurial vessels at the irradiated segment of the sciatic nerve (Fig. 2A). Thrombosis could also be demonstrated in endoneurial vessels immediately beneath the irradiation site (Fig. 2B). In contrast, endoneurial vessels in the more central region of the nerve fascicle were patent. Endothelial cells of the epineurial vessel were severely damaged with formation of vacuoles. The components of thrombi included red blood cells and platelets (Fig. 3A). Immediately after laser irradiation, myelinated and unmyelinated axons appeared normal in the central region of the nerve fascicle away from the subepineurial region (Fig. 3B). For further demonstration that axons of the sciatic nerve were not directly damaged by the laser irradiation, amplitudes of compound muscle action potential (CMAP) of the plantar muscle were assessed before and $30 \mathrm{~min}$ after laser irradiation. Amplitudes of CMAP before and $30 \mathrm{~min}$ after laser irradiation on the operated side were $2.42 \pm 0.09 \mathrm{mV}$ versus $2.46 \pm 0.07$ $\mathrm{mV}$, respectively $(P=0.82)$.

\section{Behavioral observations after laser irradiation}

After laser irradiation, rats exhibited significant changes in behaviors, gait, and stance, including guarding of the affected paw from touching the floor. The most pronounced behavioral changes and abnormal walking patterns occurred during PODs 7-14, and gradually disappeared after POD 28. Rats usually elevated the hind paw of the operated side particularly when walking on uneven surfaces beginning from POD 7 (Fig. 4). They shifted their body weight to the control side while sitting or lying. When they placed the affected foot on the floor, only the medial side of the paw touched the floor. When lying down, the operated hind paw was placed over the scrotum or tail most of the time. Sometimes spontaneous shaking of the operated hind paw was noted. 

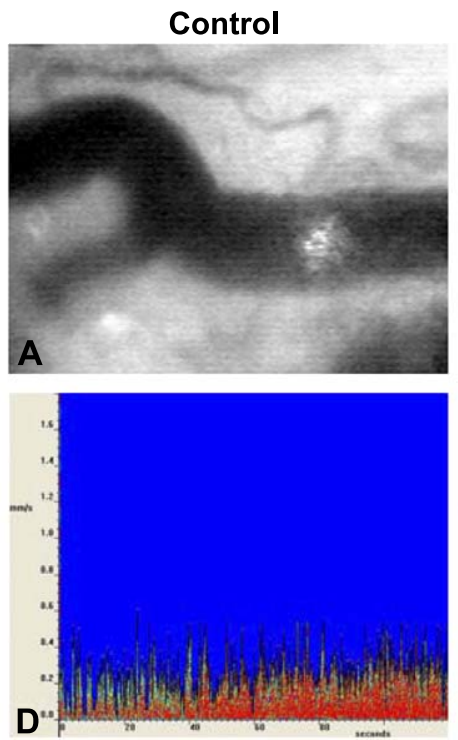
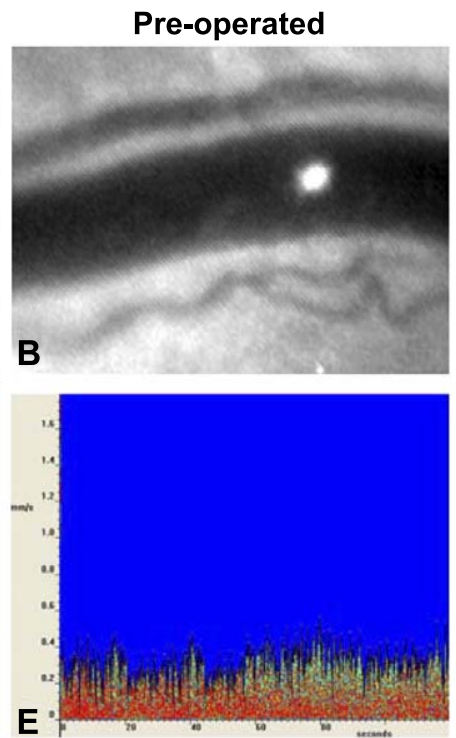
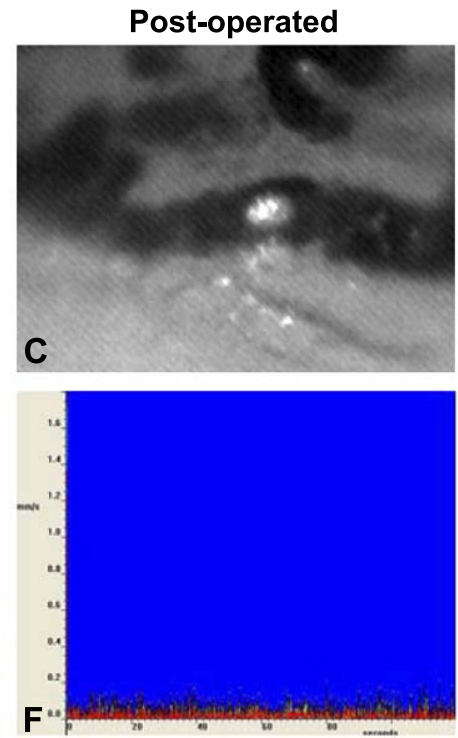

Fig. 1. Changes in epineurial arteries and blood flow after laser irradiation. (A) The appearance of epineurial arteries was smooth after dissecting free from the surrounding connective tissues on the control side. The white spot represents the reflection of the laser probe for measuring blood flow. (B) On the operated side, the exterior of the epineurial vessel was smooth before application of the laser. (C) Three minutes after laser irradiation, the outline of epineurial vessels became irregular, and the diameter was reduced. (D) The blood flow on the control side after sham operation $(0.27 \pm 0.02 \mathrm{~mm} / \mathrm{s})$. (E) The blood flow before laser irradiation was $0.30 \pm 0.04 \mathrm{~mm} / \mathrm{s}$. (F) The blood flow $3 \mathrm{~min}$ after laser irradiation was significantly reduced $(0.05 \pm 0.01 \mathrm{~mm} / \mathrm{s}, P=0.0025)$.

Beginning from POD 2, all toes were flexed together, and there was marked plantar flexion of the hind paw during walking. A limping gait gradually developed on POD 7, and the stance phase of the affected hind limb became shortened. Rats usually walked on toes of the irradiated side with the heel and footpads only briefly touching the floor.
Despite the above observations, rats appeared normal and showed no signs of stress, such as struggling and vocalization when brought to the examination rooms for the thermal and mechanical tests. The fur of the animals was well groomed, and there were no trophic changes in the four limbs. No autotomy was detected.
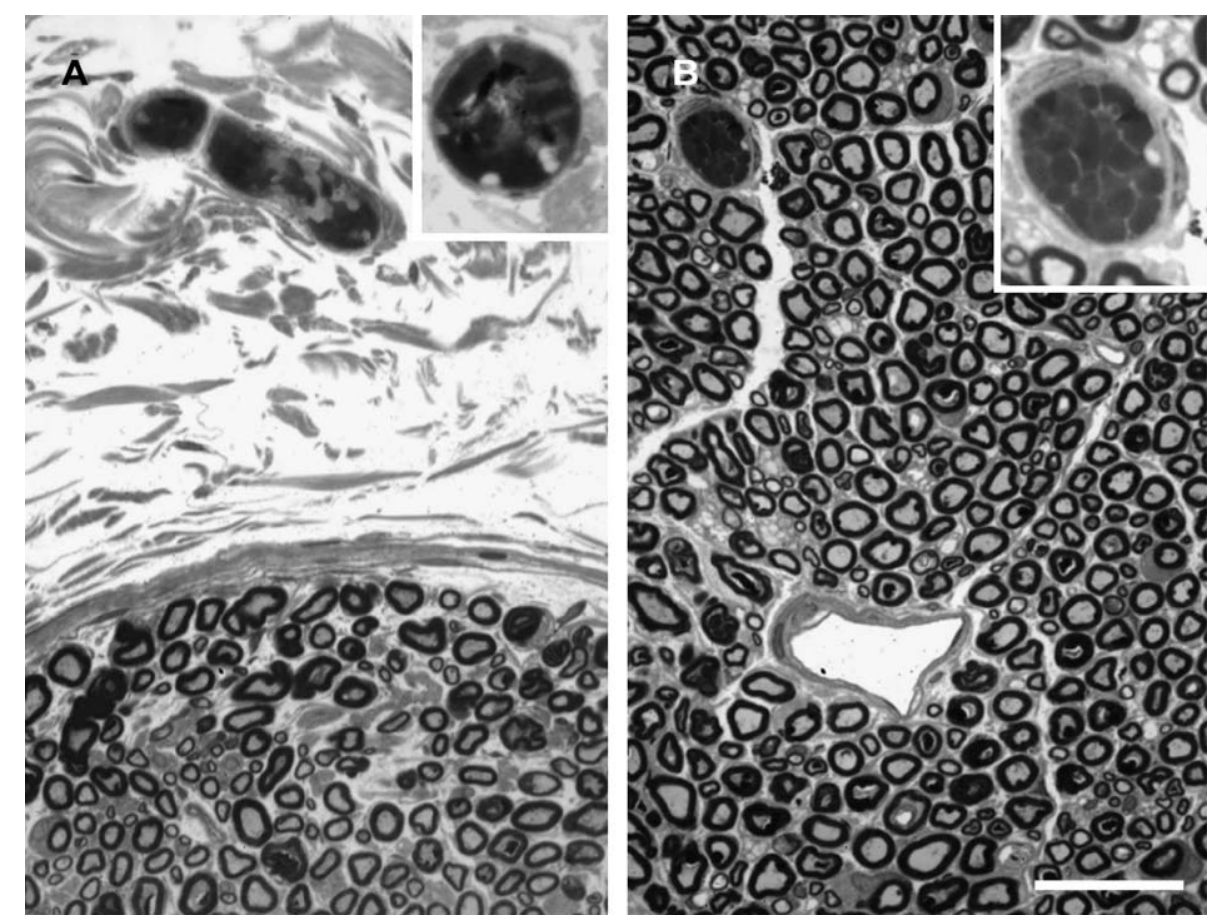

Fig. 2. Formation of thrombi $5 \mathrm{~min}$ after laser irradiation. (A) Five minutes after laser irradiation, thrombi (inset) were detected in epineurial vessels in the irradiated segment of the sciatic nerve. Only a small portion of myelinated axons beneath the perineurium became condensed, which might reflect the acute thermal effect of laser injury. Myelinated axons in the more central portion of the subepineurial region appeared intact. (B) Thrombi (inset) could also be demonstrated in one endoneurial vessel in the subepineurial region immediately beneath the irradiation site. Other endoneurial vessels in the more central region of the nerve fascicle away from the subepineurial region were patent. (Bar, $40 \mu \mathrm{m}$ in $\mathrm{A}$ and $\mathrm{B} ; 20 \mu \mathrm{m}$ for inset). 

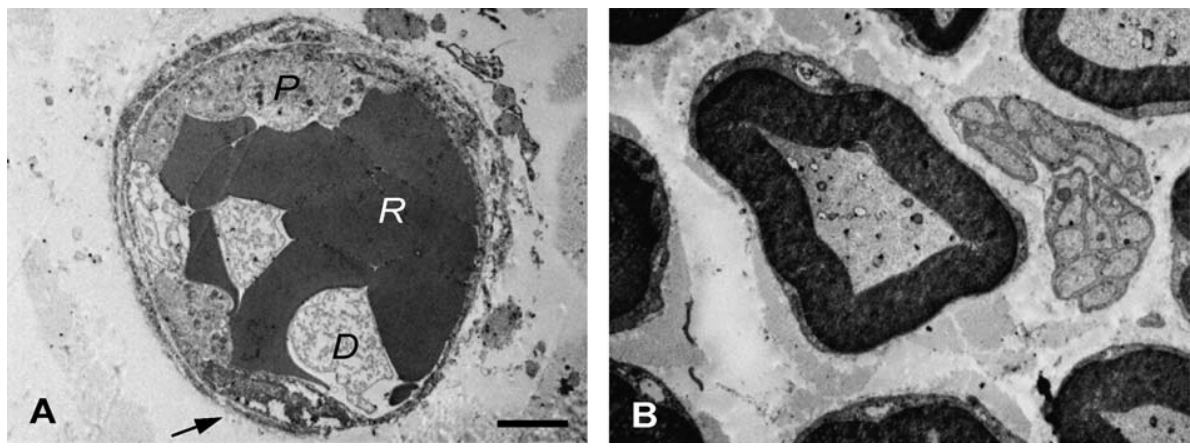

Fig. 3. Electron micrographs in the irradiated segment of the sciatic nerve $5 \mathrm{~min}$ after laser irradiation. (A) The formation of thrombi was noted in epineurial vessels $5 \mathrm{~min}$ after laser irradiation. Endothelial cells of the epineurial vessel were severely damaged with formation of vacuoles (arrow). The components of the thrombi included red blood cells (R), and granulated (P) and degranulated (D) platelets. (B) Immediately after laser irradiation, myelinated and unmyelinated axons appear normal in the central region of the nerve fascicle. (Bar, $2 \mu \mathrm{m}$ in A and B).

\section{Thermal hyperalgesia}

Rats showed significant thermal hyperalgesia beginning from POD 2, which lasted for 42 days (Fig. 5). Differences in withdrawal latencies between the irradiated side and the control side of each rat were used to assess thermal hyperalgesia, with the baseline values clustering around $0(0.39 \pm 0.24 \mathrm{~s}, P=0.1138$, Fig. 5A). After laser irradiation, differences in the withdrawal latencies became negative from POD $2(-2.95 \pm 0.41 \mathrm{~s}, P<$ $0.001)$ and reached the most negative value on POD $7(-3.16 \pm$ $0.19 \mathrm{~s}, P<0.001)$. This value remained significantly lower than the baseline value until POD $42(-1.56 \pm 0.43 \mathrm{~s}, P<0.05)$.

To quantitatively describe pain-related behaviors during thermal stimulation, we analyzed the behaviors by measuring elevation time (Fig. 5B) and calculating total behavior scores of the affected hind paws (Fig. 5C). Rats always elevated the hind foot transiently. After laser irradiation, rats elevated the affected limb for a longer period after sensing the noxious heat. The hind paw elevation time significantly increased compared to the baseline value starting from POD $4(6.50 \pm 1.20 \mathrm{~s}, P<0.05)$, and this value reached a peak on POD $21(9.15 \pm 2.84 \mathrm{~s}, P<0.05)$. The elevation time of the operated hind paws returned to the baseline level after POD 35.

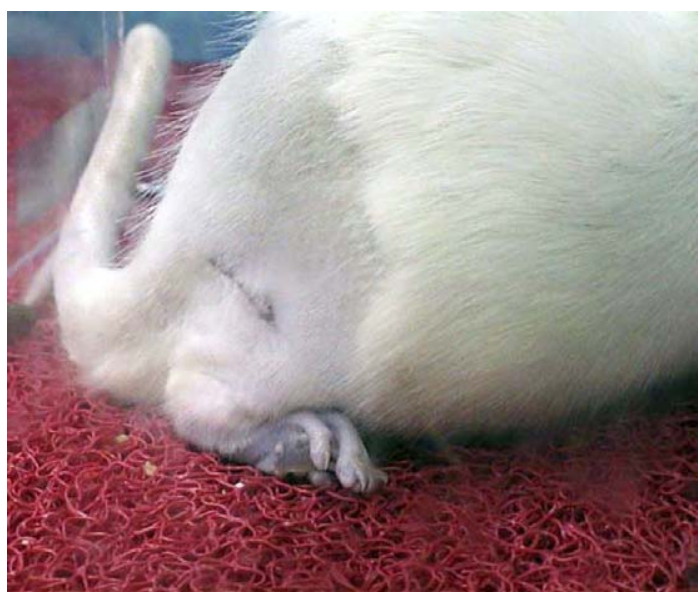

Fig. 4. Posture change after laser irradiation. The typical posture includes mild elevation of the hind paw and flexion of the toes on the operated side particularly when walking on uneven surfaces.
In addition, there were robust changes in the reaction to noxious heat after the surgery; animals usually kept the operated hind paw lifted with licking it. The behavior scores increased from POD 2 $(1.90 \pm 0.17, P<0.05)$ and reached the highest value on POD 21 $(2.43 \pm 0.31, P<0.05)$. Taken together, thermal hyperalgesia lasted for 3 weeks and gradually returned to the baseline level after POD 35 .

\section{Mechanical allodynia}

The laser irradiation injury also induced mechanical allodynia in addition to thermal hyperalgesia (Fig. 6). Mechanical thresholds were nearly identical before surgery, with the logarithm of the threshold ratio around $0(0.06 \pm 0.04, P=0.19)$. The withdrawal thresholds to mechanical stimuli were significantly reduced starting from POD $4(-0.44 \pm 0.08, P<0.05)$. Mechanical allodynia was significant up to POD $21(-0.51 \pm$ $0.16, P<0.05)$. Withdrawal thresholds returned to the baseline value after POD 28.

Neuropathology of myelinated nerves in laser-induced neuropathy

To understand the degree of damage to myelinated nerve fibers after laser irradiation, we examined semi-thin sections of the sciatic nerves on POD 7. There were three zones of nerve injuries in the irradiated segment from the subepineurial region to the central part of the nerve fascicle (Fig. 7A). Degenerating axons with debris were scattered in the area immediately beneath the epineurium (* in Fig. 7A, B). Next to this region was an area containing demyelinating axons (** in Fig. $7 \mathrm{~A}, \mathrm{C})$. An increase in the extracellular space between axons was noted in the region peripheral to the demyelination zone, and some demyelinating axons could still be found in this region (*** in Fig. 7A, D). There was only endoneurial edema or minimal nerve pathology in the area opposite the site of laser irradiation. In the sciatic nerve distal to the irradiation site, the loss of myelinated fibers was observed in one area of the sciatic nerve with intact axons in the other part of the sciatic nerve (Fig. 8).

Changes in compound muscle action potentials (CMAPs) of the sciatic nerves after laser irradiation

To investigate the effect of motor nerve damage after laser irradiation, we measured the amplitudes of CMAPs of the sciatic 
A

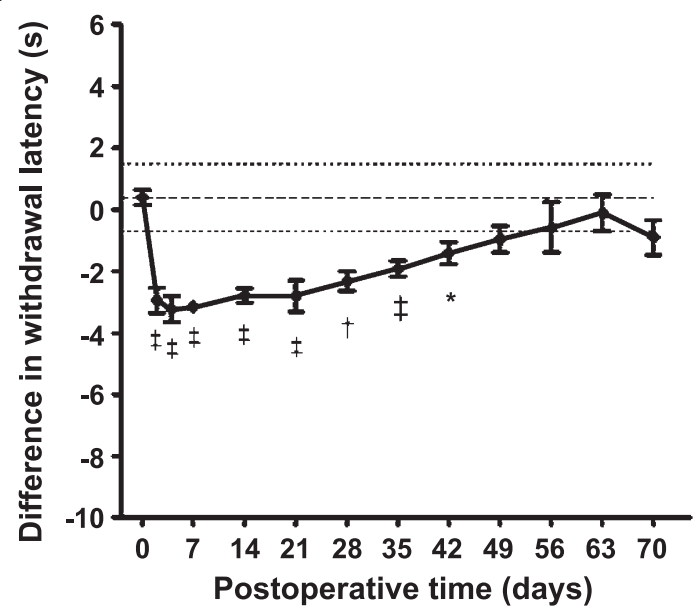

B

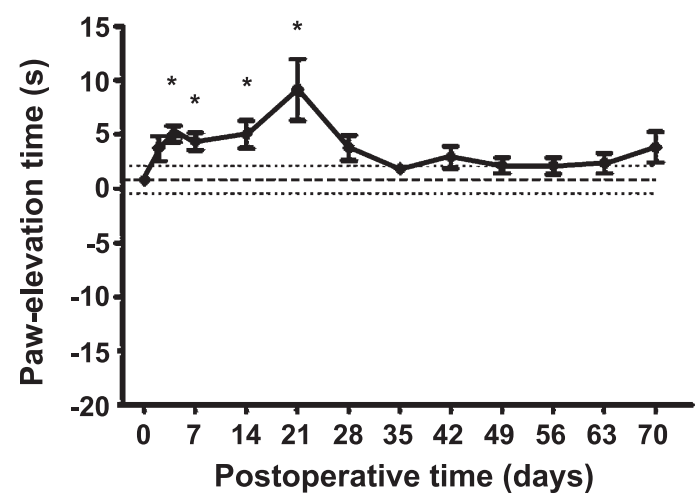

C

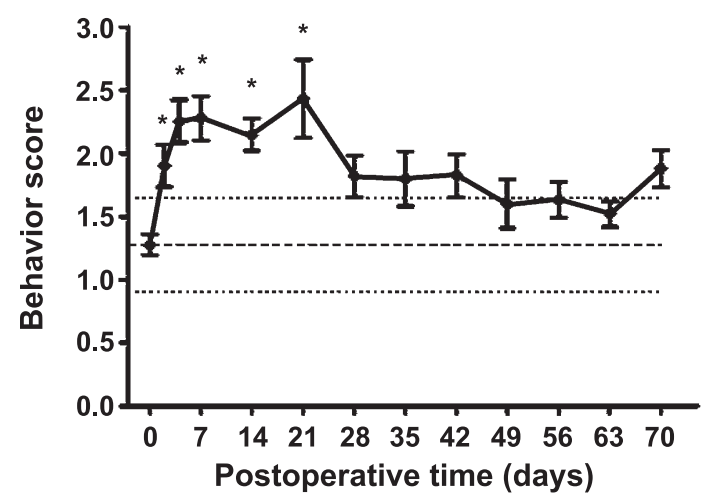

Fig. 5. Temporal course of thermal hyperalgesia and quantitative changes in behaviors on the thermal test in laser-induced painful neuropathy. (A) Differences in the withdrawal latencies in each rat were used to assess thermal hyperalgesia. Rats showed significant thermal hyperalgesia beginning from postoperative days (PODs) 2 to 42. (B) Behavioral changes during thermal stimulation were assessed quantitatively by hind paw elevation time. This parameter significantly increased from PODs 4 to 21 . (C) Behavioral changes during thermal stimulation were quantitatively evaluated by behavior scores. Significant behavioral changes were observed from PODs 2 to 21. Dashed lines indicate the mean value of the preoperative test, and dotted lines represent the range of $\mathrm{SD}$. ${ }^{*} P<0.05$; $\dagger P<0.01 ; \ddagger P<0.001$

nerves. There was significant reduction in the amplitudes of CMAPs after laser irradiation (Fig. 9A). Before surgery, the amplitude of CMAP was $2.46 \pm 0.04 \mathrm{mV}$. On POD 7, this value was reduced to $1.60 \pm 0.09 \mathrm{mV}, 65.0 \%$ of the control side (Fig. 9B) $(P<0.0001)$.

Changes in sensory nerve action potentials (SNAPS) of the sural nerves after laser irradiation

To evaluate the damage to large-diameter sensory nerves, amplitudes of SNAPs of the sural nerves were recorded on POD 7 (Fig. 10A). The amplitude of SNAP on the operated side was significantly reduced compared to that on the control side (71.00 \pm $15.55 \mu \mathrm{V}$ vs. $167.10 \pm 17.99 \mu \mathrm{V}, P=0.0043$, Fig. $10 \mathrm{~B})$, about $42.5 \%$ of the value on the control side.

\section{Reduced motor innervation of plantar muscles after laser irradiation}

We further examined structural changes in NMJs of plantar muscles after laser irradiation. Motor unnervation was demonstrated by combined cholinesterase histochemistry and immunohistochemistry with PGP 9.5 (Fig. 11). NMJs on the control side were abundantly innervated by PGP $9.5(+)$ axons (Fig. 11A), and these axons extended into the entire NMJ (Fig. 11B). On POD 7 of laser irradiation, some NMJs of the plantar muscle on the operated side became denervated. NMJs at some areas of the plantar muscle were innervated normally (Figs. 11C-D), but NMJs at other parts of the muscle were denervated with axonal debris (Figs. 11E-F).

The ratios of innervated NMJs on both sides were calculated for quantifying the degree of damage to motor nerve terminals. This ratio on the operated side on POD 7 was significantly reduced compared to that on the control side $(71.2 \% \pm 1.7 \%$ vs. $93.3 \% \pm$ $2.0 \%, P=0.0002$ ), about $76.3 \%$ of the control value (Fig. 12).

Skin innervation in the territory of the sciatic nerve after laser irradiation

To evaluate the damage to small-diameter sensory nerve terminals in the skin, we performed immunohistochemical analyses on footpads of the hind paw innervated by the sciatic nerve and quantified epidermal nerve densities.

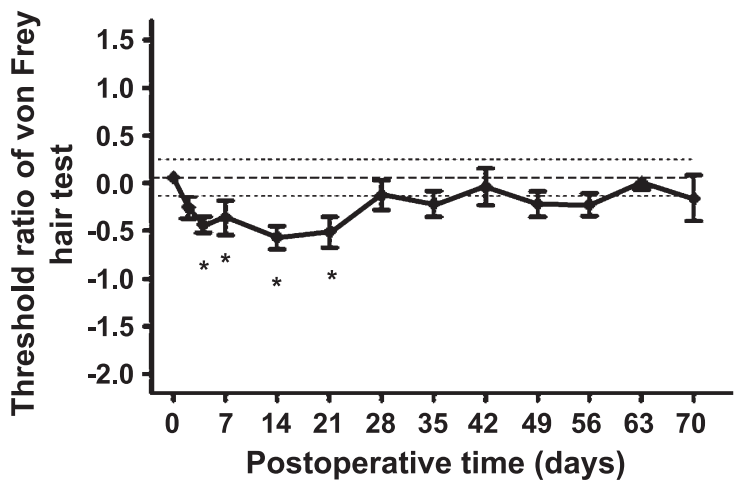

Fig. 6. Temporal course of mechanical allodynia in laser-induced painful neuropathy. The logarithm of ratio of mechanical thresholds to von Frey hair test (operated side/control side) was used as the index of mechanical allodynia. The values were significantly decreased from postoperative days (PODs) 4 to 21. This value returned to the baseline level after POD 28 . Dashed lines indicate the mean value of the preoperative test, and dotted lines represent the range of SD. $* P<0.05$. 

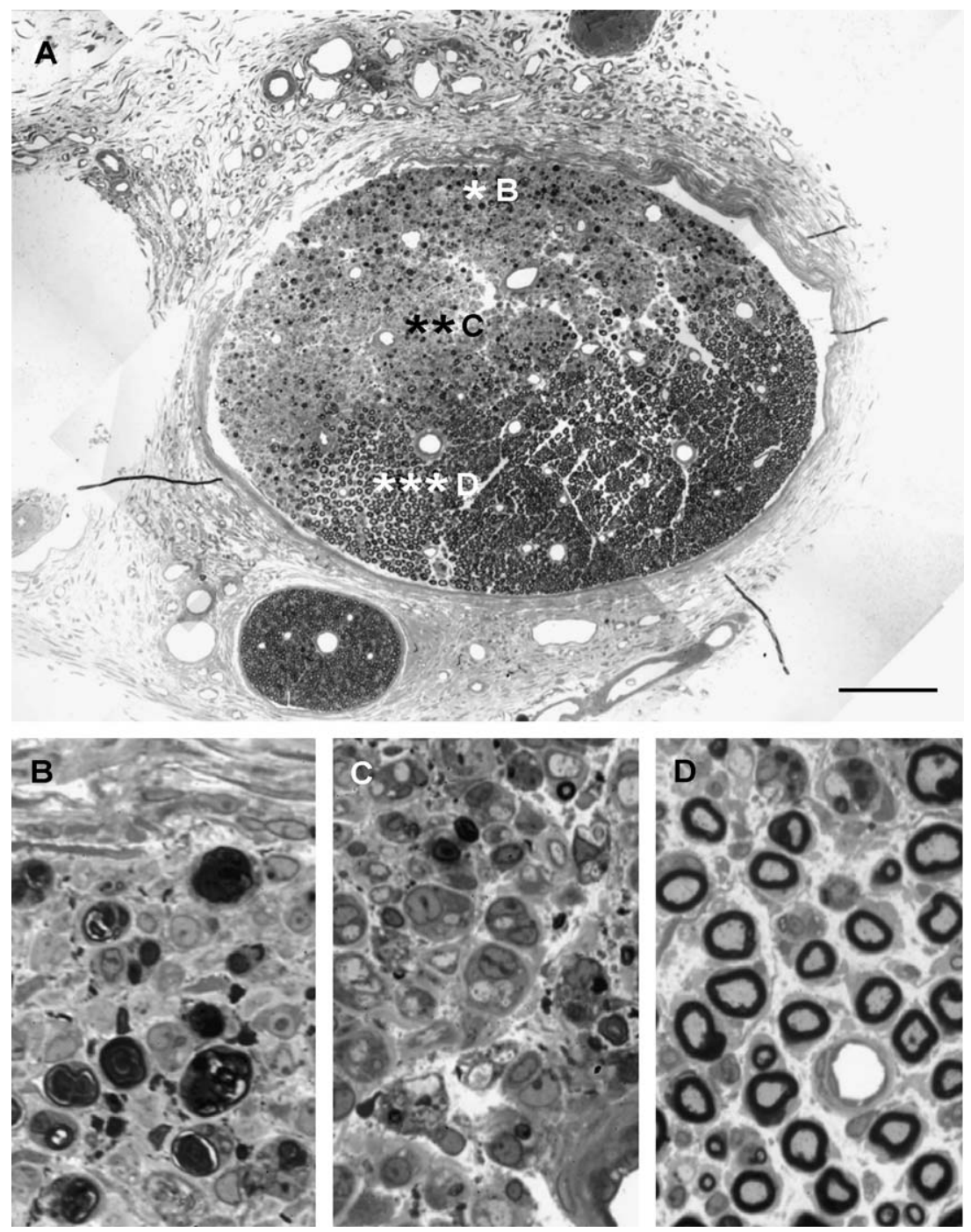

Fig. 7. Myelinated fiber loss in the irradiated segment of the sciatic nerve after laser irradiation. (A) On postoperative day (POD) 7, there were three regions with different patterns of nerve injury (labeled as * in $\mathrm{B}, * *$ in $\mathrm{C}$, and $* * *$ in $\mathrm{D}$ ). (B) Axonal degeneration was the prominent feature in the subepineurial region directly exposed to laser irradiation. (C) Demyelinating axons can be noted in the region peripheral to the area containing degenerating axons. (D) Endoneurial edema became apparent in the region next to the demyelination zone. (Bar, $160 \mu \mathrm{m}$ in A; $20 \mu \mathrm{m}$ in B-D).

The epidermis of the control side was abundantly innervated by PGP 9.5(+) fibers (Fig. 13A). These nerves originated from the subepidermal nerve plexus, penetrated through the epidermal-dermal junction and traveled perpendic- ularly in the epidermis. The epidermal nerve density was $10.28 \pm 0.73$ fibers $/ \mathrm{mm}$ on the control side (Fig. 14A). On POD 7, the PGP $9.5(+)$ fibers on the operated side were significantly reduced (Fig. 13B), with an epidermal nerve
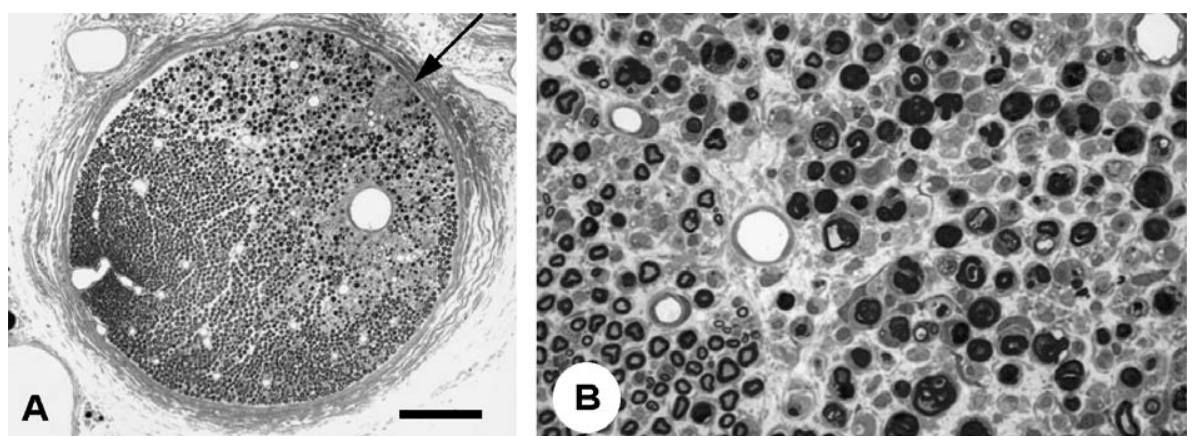

Fig. 8. Focal loss of myelinated fibers in the sciatic nerve distal to the irradiation site on postoperative day 7. (A) A low-power view of the sciatic nerve showing focal loss of myelinated nerves in one portion of the sciatic nerve (arrow). (B) High-power image demonstrating the boundary transition from the region containing normal axons to the area containing degenerating nerves (right portion). (Bar, $160 \mu \mathrm{m}$ in A; $20 \mu \mathrm{m}$ in B-D). 
A

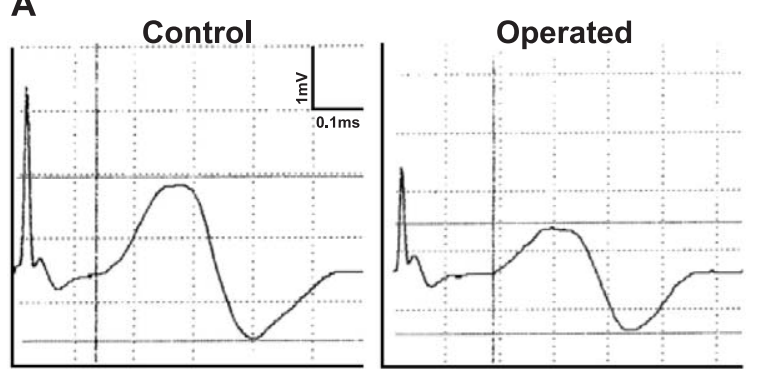

B

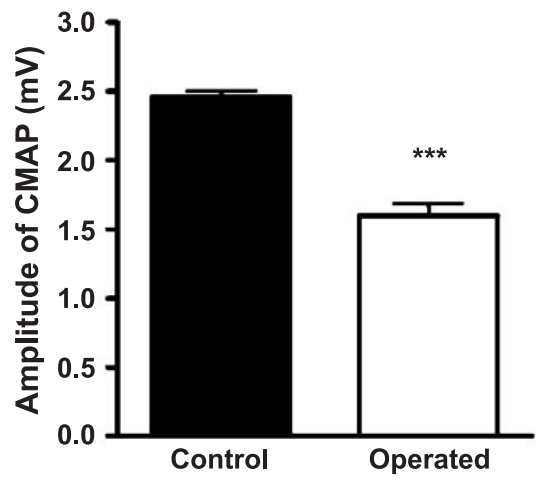

Fig. 9. Changes in nerve conduction studies after laser irradiation. (A) Tracings of compound muscle action potentials (CMAPs) on the control (left) and operated (right) sides on postoperative day (POD) 7. (B) Amplitudes of CMAPs were significantly reduced on POD 7 (2.50 \pm 0.04 vs. $1.71 \pm 0.08 \mathrm{mV}) .{ }^{*} P<0.001$

density of $5.46 \pm 1.63$ fibers $/ \mathrm{mm}, 53.11 \%$ of the control side $(P<0.05)$ (Fig. 14A).

Similar to PGP 9.5(+) fibers, CGRP $(+)$ fibers and $\mathrm{SP}(+)$ fibers were found in the epidermis of the control side (Figs. 13C, E) with epidermal nerve densities of $4.42 \pm 0.40$ and $2.14 \pm 0.24$ fibers/mm, respectively (Figs. 14B, C). On POD 7 after laser irradiation, $\mathrm{CGRP}(+)$ fibers on the operated side were reduced to $2.20 \pm 0.61$ fibers $/ \mathrm{mm},(P<0.05), 49.77 \%$ of the control side (Figs. 13D, 14B). SP $(+)$ fibers on the operated side had decreased to $0.90 \pm 0.42$ fibers $/ \mathrm{mm}(P<0.05), 42.06 \%$ of the control side (Figs. 13F, 14C). The difference in epidermal nerve density of GAP 43(+) fibers between the operated side and the control side was not statistically significant $(5.75 \pm 1.15$ vs. $5.84 \pm 0.78$ fibers $/ \mathrm{mm}, P=0.22$ ) (Figs. $13 \mathrm{G}, \mathrm{H}$, and $14 \mathrm{D}$ ).

\section{Discussion}

\section{Experimental systems of neuropathic pain}

This report documents a new experimental pain-inducing system of focal neuropathy by using brief laser irradiation alone without photosensitive dyes. The present study demonstrates that the nerve injury produced by such a simple approach can induce neuropathic pain in addition to laser-induced ischemia (Rosen et al., 2001). Characteristic manifestations include spontaneous and nociception-evoked pain behaviors, thermal hyperalgesia, and mechanical allodynia similar to other neuropathic pain systems induced by mechanical injury as summarized in Table 2 . There are certain unique features in the current approach compared with photochemical systems. First, the durations of neuropathic pain, including mechanical allodynia and thermal hyperalgesia, were shorter than with the other types; thermal hyperalgesia lasted for 5 weeks in the current system, compared to 2-3 months with the photochemical approach (Hao et al., 2000; Kupers et al., 1998) and mechanical injury (Bennett and Xie, 1988; Kupers et al., 1992; Malmberg and Basbaum, 1998). Several factors may account for these differences. For example, in the current approach, the effect of laser irradiation was limited to one portion of the sciatic nerve fascicle, particularly, the subepineurial regions immediately underneath the site of laser irradiation. Other alternatives may include potential reversibility of nerve lesions or less extensive changes in central sensitization (Craig, 2003); confirming these possibilities requires further investigations. Nevertheless, this approach provides a simple and brief procedure to generate focally painful neuropathy.

Degree of nerve damage and magnitude of neuropathic pain behaviors

Nerve injury is an essential factor in inducing neuropathic pain, and an important issue for elucidating different mechanisms of neuropathic pain requires an animal system of neuropathic pain with quantifiable nerve injury. The present system indicates that the duration of laser irradiation could be adjusted to induce neuropathic pain. Laser irradiation of $15 \mathrm{~s}$ produced the lowest success rate of neuropathic pain lasting for only 1 week. In contrast, laser irradiation for $60 \mathrm{~s}$ resulted in a combination of thermal hyperalgesia and anesthesia. The result extends previous

A

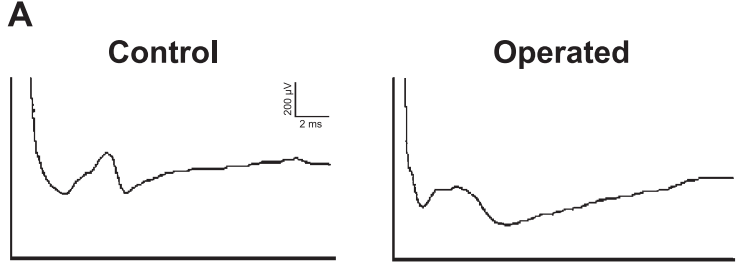

B

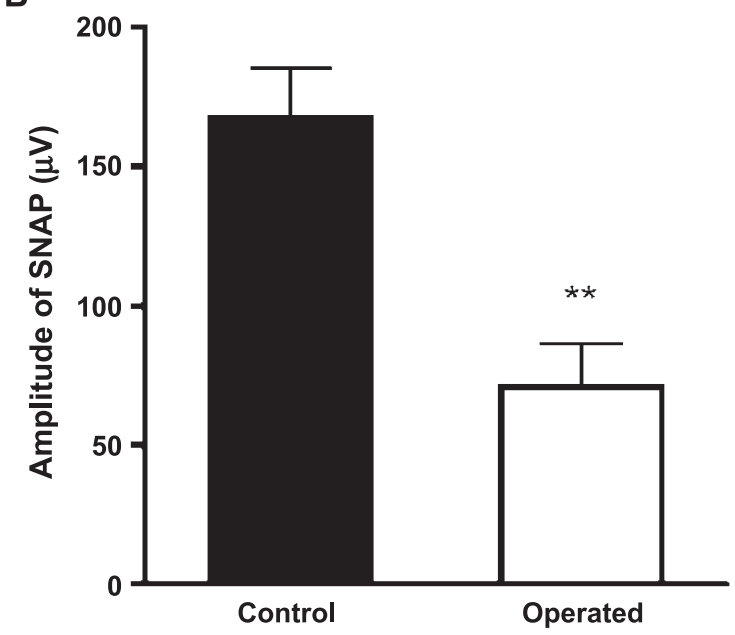

Fig. 10. Changes in the amplitudes of sensory nerve potentials (SNAPs) 7 days postoperatively after laser irradiation. (A) Representative tracings of sensory nerve action potentials (SNAPs) on the control side (left) and on the operated side (right). (B) Amplitudes of SNAPs were significantly decreased on postoperative day 7 after laser irradiation $(167.10 \pm 17.99$ vs. $71.00 \pm 15.55 \mu \mathrm{V}) . * * P<0.01$. 

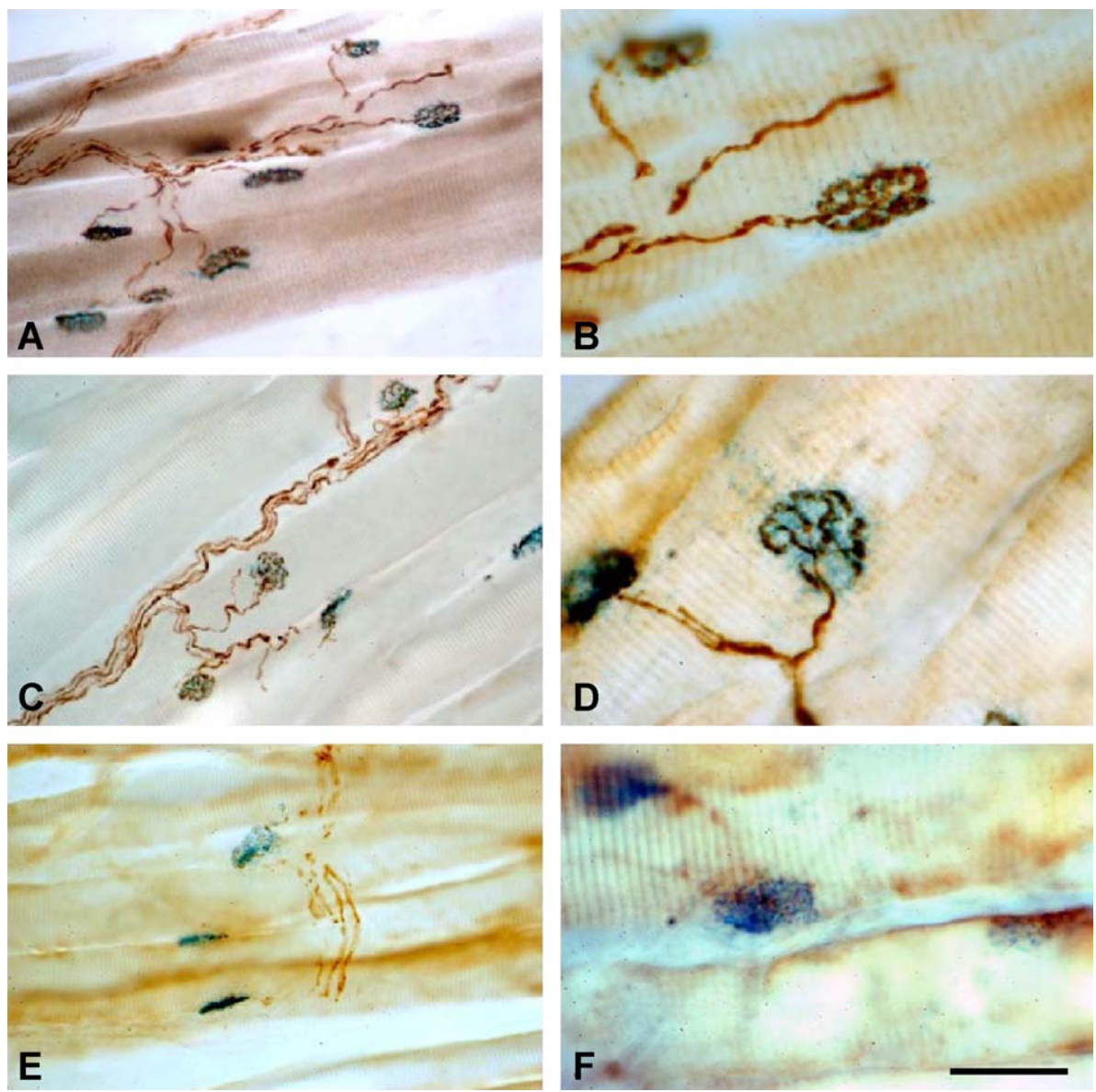

Fig. 11. Denervation of neuromuscular junctions (NMJs) in plantar muscles 7 days after laser irradiation. Sections of the plantar muscles were stained with cholinesterase for NMJs (blue) and immunohistochemistry for the axonal marker, protein gene product, PGP 9.5 (brown). A and B were photographs from control sides, and C-F were from operated sides. (A) NMJs were abundantly innervated by PGP $9.5(+)$ fibers on the control side. (B) PGP $9.5(+)$ axons were in branches of the NMJ at higher magnification. (C) NMJs at some areas of the plantar muscles on the operated side were innervated after laser irradiation. (D) PGP 9.5(+) nerves were in NMJs from (C). (E) At some areas of the plantar muscle, NMJs were completely denervated. (F) No PGP 9.5(+) fibers were found in a NMJ at a higher magnification from E. (Bar, $50 \mu \mathrm{m}$ in A, C, E; $20 \mu \mathrm{m}$ in B, D, F).

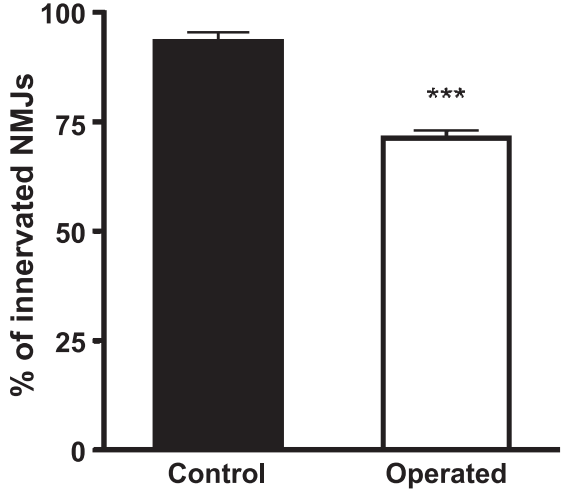

Fig. 12. Changes in the ratio of the innervated neuromuscular junctions (NMJs) on postoperative day 7 after laser irradiation. The combined staining of cholinesterase histochemistry and immunohistochemistry for protein gene product 9.5 was used to label NMJs and axons, respectively. Ratios of the innervated NMJs were calculated on the control and the operated sides. The ratio of innervated NMJs on the operated side was significantly decreased compared to that on the control side 7 days after laser irradiation. $* * * P<0.001$. studies on freezing injury-induced neuropathic pain, in which the magnitude and duration of the hyperalgesia were related to the extent of nerve damage (Myers et al., 1996; Wagner et al., 1995). Several lines of evidence have indicated that the injured area in the sciatic nerve is proportional to the magnitude of the insults applied to the nerve. In studies using the $\mathrm{CO}_{2}$ laser, the stronger the laser energy used to irradiate the sciatic nerve, the greater nerve damage that was noted (Menovsky et al., 1996; Myers et al., 1985). However, it has not been demonstrated that these $\mathrm{CO}_{2}$ laserinduced nerve injuries could cause neuropathic pain (Menovsky et al., 1996, 2000; Myers et al., 1985). Thus the dose of laser energy is an adjustable factor, so that the degree of nerve injury and the extent of neuropathic pain behaviors can be predicted. The current approach therefore is useful in determining relationships among nerve injury, pain behaviors, and underlying mechanisms.

\section{Neuropathological effects of laser irradiation and vulnerability of myelinated versus unmyelinated nerves to laser irradiation}

Laser-induced nerve injury can be classified into two phases: an immediate phase and a late phase. The immediate thermal effect of 

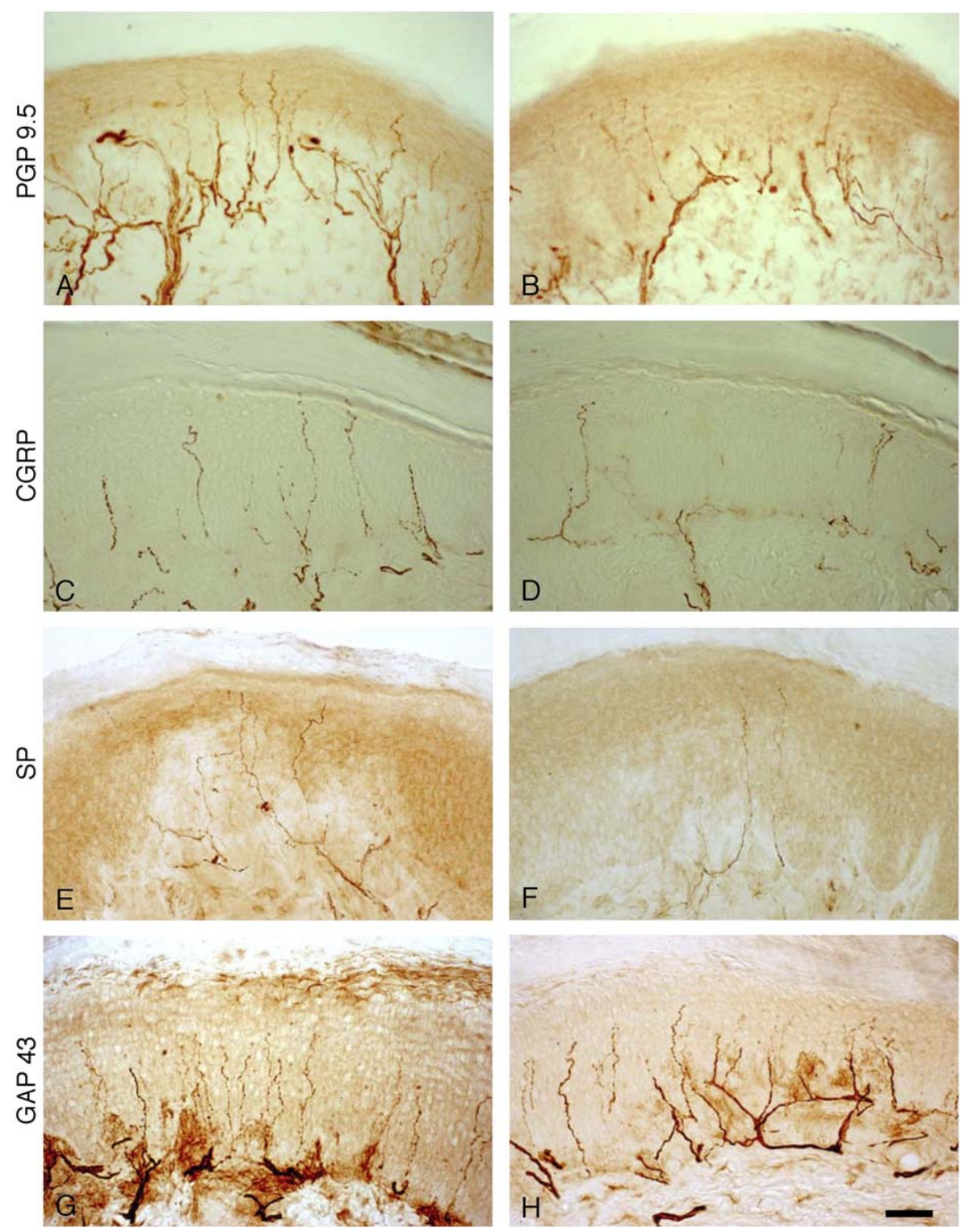

Fig. 13. Skin innervation of the hind paws from the control side (A, C, E, and G) and the operated side (B, D, F, and H). Neuronal markers include protein gene product 9.5 (PGP 9.5, in A and B), calcitonin gene-related peptide (CGRP, in C and D), substance P (SP, in E and F), and growth-associated protein 43 (GAP 43, in $\mathrm{G}$ and $\mathrm{H}$ ). (A) The epidermis on the control side abundantly innervated by PGP 9.5(+) nerve fibers. (B) PGP $9.5(+)$ nerve fibers reduced on the operated side. (C) CGRP $(+)$ nerves present on the control side, although the number of CGRP $(+)$ nerves is smaller than that of PGP 9.5(+) nerves. (D) Decreased number of CGRP $(+)$ fibers after laser irradiation. (E) SP(+) nerves innervating the skin on the control side. (F) Reduced number of SP(+) fibers in the epidermis on the operated side. (G) GAP 43(+) fibers noted in the epidermis on the control side. (H) Similar abundances of GAP 43(+) fibers on the operated side and on the control side in $(\mathrm{G})$. (Bar, $30 \mu \mathrm{m}$ in $\mathrm{A}-\mathrm{H})$.

laser irradiation in the current report was limited to the subepineurial region and depended on the nature and dose of the laser. This is in contrast to the $\mathrm{CO}_{2}$ laser, which causes much more extensive thermal injury than the diode laser of $532 \mathrm{~nm}$ used in the current system (Menovsky et al., 1996, 2000); after $\mathrm{CO}_{2}$ laser irradiation on sciatic nerves, both the epineurium and endoneurium are severely damaged by the thermal effect. The entire nerve becomes edematous, and pyknotic nuclei appear in fibroblasts. All these signs indicate extensive injury by the $\mathrm{CO}_{2}$ laser, which is absorbed by tissue water and causes vaporization of tissue.

Subsequent injury was related to ischemic events as demonstrated by the formation of thrombi and reduced blood flow through epineurial vessels. Based on previous studies comparing tight ligation and chronic constriction injury, it is possible that extensive injury to the sciatic nerve will cause anesthesia instead of hyperalgesia (Lin et al., 2001). In the current system, only a limited portion of the subepineurial area was injured by laser irradiation during the acute stage, while other portions of the sciatic nerves remained intact microscopically. This approach provides an opportunity to investigate the degree of nerve injury and the occurrence of neuropathic pain.

The vulnerability of large-diameter versus small-diameter nerves in painful neuropathy is an intriguing issue (Basbaum et al., 1991; Gautron et al., 1990; Guilbaud et al., 1993; Nuytten et al., 1992); this issue is complicated because ischemic insults and mechanical injuries can generate various outcomes (Parry and 
A

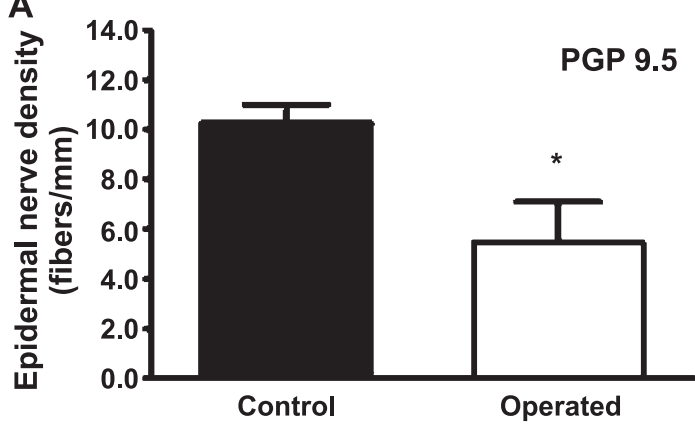

C
곰
B

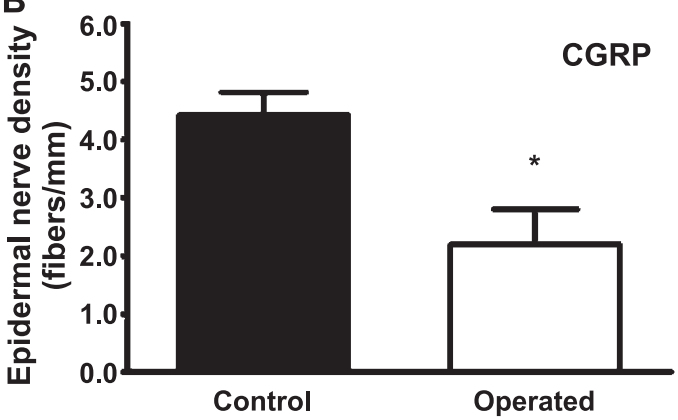

D

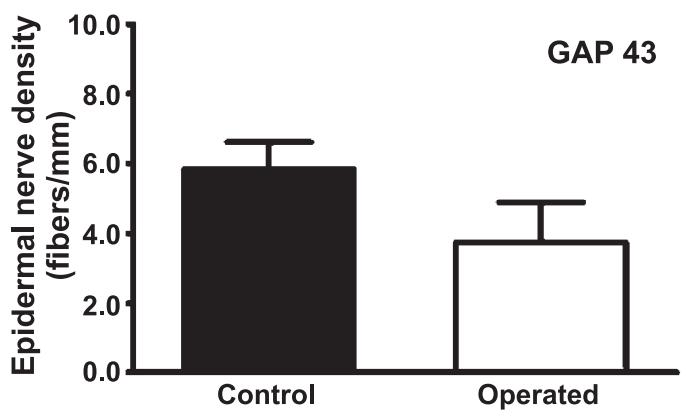

Fig. 14. Changes in epidermal density (END) on postoperative day 7 after laser-induced painful neuropathy. Quantitative comparison of ENDs based on immunohistochemical staining in Fig. 10 is plotted. ENDs of protein gene product 9.5 (PGP 9.5, in A), calcitonin gene-related peptide (CGRP, in B), and substance P (SP, in C) on the operated sides were significantly reduced compared to those on the control side. ENDs of growth-associated protein 43 (GAP 43 , in D) are similar between the control and operated sides. ${ }^{*} P<0.05$.

Brown, 1982; Vital et al., 1986). The extent of nerve damage by laser irradiation appears differentially, including large-diameter versus small-diameter nerve fibers, and different phenotypes of sensory nerve terminals in the skin. Epidermal nerves of PGP 9.5, CGRP, and SP phenotypes were reduced more prominently (42.1$53.1 \%$ of the control side) compared to large-diameter motor nerves as evaluated by the ratios of innervated NMJs ( $76.3 \%$ of the control side). This finding indicates that the damage to smalldiameter nerves was more extensive than that to large-diameter nerves in the laser-induced focal neuropathy. This is in contrast to other neuropathic pain models using the photochemical approach; myelinated axons were much more susceptible than unmyelinated axons to damage (Hao et al., 2000; Kupers et al., 1998). Differences in the extent and degree of nerve injury may underlie

Table 2

Comparison of experimental painful neuropathies

\begin{tabular}{|c|c|c|c|c|}
\hline \multirow{2}{*}{$\frac{\text { Type }}{\text { Method }}$} & \multicolumn{2}{|l|}{ Laser-induced injury } & \multicolumn{2}{|l|}{ Mechanical injury } \\
\hline & $\begin{array}{l}\text { Laser irradiation alone } \\
\text { (current study) }\end{array}$ & $\begin{array}{l}\text { Photochemical injury } \\
\text { (Kupers et al., 1998) }\end{array}$ & $\begin{array}{l}\text { PSL } \\
\text { (Seltzer et al., 1990) }\end{array}$ & $\begin{array}{l}\text { CCI } \\
\text { (Bennett and Xie, 1988) }\end{array}$ \\
\hline Laser type & Diode laser & Argon laser & No & No \\
\hline Wavelength (nm) & 532 & 514 & & \\
\hline Laser power $(\mathrm{mW})$ & 100 & 160 & & \\
\hline Irradiation time (s) & 30 & $30-60$ & & \\
\hline Photosensitive dye & No & Erythrosine B & No & No \\
\hline Spontaneous pain behaviors & Yes & Yes & Yes & Yes \\
\hline Thermal hyperalgesia & Yes & Yes & Yes & Yes \\
\hline Peak & Weeks 2 and 3 & Weeks 1 and 3 & Week 1 & Weeks 2 and 5 \\
\hline Duration & 6 weeks & 8 weeks & $>3$ months & $2-3$ months \\
\hline Mechanical allodynia & Yes & Yes & Yes & Yes \\
\hline Peak & Weeks 2 and 3 & Week 1 & Weeks 1 and 2 & Weeks 2 and 3 \\
\hline Duration & 3 weeks & 10 weeks & $>3$ months & $2-3$ months \\
\hline \multicolumn{5}{|l|}{ Pathology } \\
\hline Affected regions & Partial nerve fascicle & $\begin{array}{l}\text { Partial or entire nerve } \\
\text { fascicle }\end{array}$ & $\begin{array}{l}\text { Complete injury of the } \\
\text { affected nerve fascicle }\end{array}$ & $\begin{array}{l}\text { Partial nerve injury in } \\
\text { the entire fascicle }\end{array}$ \\
\hline Skin innervation & Reduced & NA & Reduced & Reduced \\
\hline Amplitude of CMAP & Reduced & NA & NA & NA \\
\hline
\end{tabular}

PSL indicates partial sciatic nerve ligation; CCI, chronic constriction injury; NA, not available; CMAP, compound muscle action potential of the plantar muscles on stimulation of the sciatic nerve. 
the difference. Alternatively, different spatial patterns of nerve fibers may contribute to the differential degrees of large- versus small-fiber damage.

Intriguingly, epidermal nerve fibers of various phenotypes are differentially vulnerable to laser irradiation. On human studies, skin innervation is reduced in neuropathic pain, such as painful sensory neuropathy (Holland et al., 1997; Periquet et al., 1999) and postherpetic neuralgia (Oaklander et al., 1998). In animal models of neuropathic pain, several groups including ours have demonstrated a reduction of epidermal nerve densities after partial sciatic nerve injury and chronic constriction injury (Lin et al., 2001; Lindenlaub and Sommer, 2002; Ma and Bisby, 2000). It is not clear whether different subtypes of epidermal nerves are reduced to the same extent. The reduction of epidermal nerves in the current model not only extends previous observations of skin denervation with neuropathic pain, but also provides additional information regarding the susceptibility of epidermal nerves of different phenotypes to laser irradiation. The reduction in GAP 43(+) nerves was minimal, while epidermal nerves positive for PGP 9.5, CGRP, and SP were reduced with laser-induced neuropathic pain. This is in contrast to mechanical injury, in which epidermal nerves of different phenotypes were damaged to a similar degree (Lin et al., 2001; Lindenlaub and Sommer, 2002). These results suggest that partial injury is required to produce neuropathic pain and that epidermal nerves of different phenotypes are susceptible to different types of neuropathic pain models.

\section{Acknowledgment}

This work was supported by the National Health Research Institute, Taiwan (NHRI-EX92-9021NL, NHRI-EX93-9323NI).

\section{References}

Basbaum, A.I., Gautron, M., Jazat, F., Mayes, M., Guilbaud, G., 1991. The spectrum of fiber loss in a model of neuropathic pain in the rat: an electron microscopic study. Pain 47, 359-367.

Bennett, G.J., Xie, Y.K., 1988. A peripheral mononeuropathy in rat that produces disorders of pain sensation like those seen in man. Pain 33, $87-107$.

Chaplan, S.R., Bach, F.W., Pogrel, J.W., Chung, J.M., Yaksh, T.L., 1994. Quantitative assessment of tactile allodynia in the rat paw. J. Neurosci. Methods 53, 55-63.

Craig, A.D., 2003. Pain mechanisms: labeled lines versus convergence in central processing. Annu. Rev. Neurosci. 26, 1-30.

Decosterd, I., Woolf, C.J., 2000. Spared nerve injury: an animal model of persistent peripheral neuropathic pain. Pain 87, 149-158.

Dolmans, D.E., Fukumura, D., Jain, R.K., 2003. Photodynamic therapy for cancer. Nat. Rev., Cancer 3, 380-387.

Doubell, T.P., Woolf, C.J., 1997. Growth-associated protein 43 immunoreactivity in the superficial dorsal horn of the rat spinal cord is localized in atrophic $\mathrm{C}$-fiber, and not in sprouted A-fiber, central terminals after peripheral nerve injury. J. Comp. Neurol. 386, 111-118.

Gautron, M., Jazat, F., Ratinahirana, H., Hauw, J.J., Guilbaud, G., 1990. Alterations in myelinated fibres in the sciatic nerve of rats after constriction: possible relationships between the presence of abnormal small myelinated fibres and pain-related behaviour. Neurosci. Lett. 111, $28-33$.

Gazelius, B., Cui, J.G., Svensson, M., Meyerson, B., Linderoth, B., 1996. Photochemically induced ischaemic lesion of the rat sciatic nerve. A novel method providing high incidence of mononeuropathy. NeuroReport 7, 2619-2623.
Guilbaud, G., Gautron, M., Jazat, F., Ratinahirana, H., Hassig, R., Hauw, J.J., 1993. Time course of degeneration and regeneration of myelinated nerve fibres following chronic loose ligatures of the rat sciatic nerve: can nerve lesions be linked to the abnormal pain-related behaviours? Pain 53, 147-158.

Hao, J.X., Blakeman, K.H., Yu, W., Hultenby, K., Xu, X.J., WiesenfeldHallin, Z., 2000. Development of a mouse model of neuropathic pain following photochemically induced ischemia in the sciatic nerve. Exp. Neurol. 163, 231-238.

Hargreaves, K., Dubner, R., Brown, F., Flores, C., Joris, J., 1988. A new and sensitive method for measuring thermal nociception in cutaneous hyperalgesia: a new and sensitive method for measuring thermal nociception in cutaneous hyperalgesia. Pain 32, 77-88.

Hegde, A.N., Inokuchi, K., Pei, W., Casadio, A., Ghirardi, M., Chain, D.G., Martin, K.C., Kandel, E.R., Schwartz, J.H., 1997. Ubiquitin C-terminal hydrolase is an immediate-early gene essential for long-term facilitation in Aplysia. Cell 89, 115-126.

Hilliges, M., Wang, L., Johansson, O., 1995. Ultrastructural evidence for nerve fibers within all vital layers of the human epidermis. J. Invest. Dermatol. 104, 134-137.

Holland, N.R., Stocks, A., Hauer, P., Cornblath, D.R., Griffin, J.W., McArthur, J.C., 1997. Intraepidermal nerve fiber density in patients with painful sensory neuropathy. Neurology 48, 708-711.

Hsieh, S.T., Chiang, H.Y., Lin, W.M., 2000. Pathology of nerve terminal degeneration in the skin. J. Neuropathol. Exp. Neurol. 59, $297-307$.

IASP Committee, 1980. Ethical standards for investigations of experimental pain in animals. The committee for research and ethical issues of the International Association for the Study of Pain. Pain 9, $141-143$.

Iida, H., Schmelzer, J.D., Schmeichel, A.M., Wang, Y., Low, P.A., 2003. Peripheral nerve ischemia: reperfusion injury and fiber regeneration. Exp. Neurol. 184, 997-1002.

Kennedy, W.R., Said, G., 1999. Sensory nerves in skin: answers about painful feet? Neurology 53, 1614-1615.

Ko, M.H., Chen, W.P., Lin-Shiau, S.Y., Hsieh, S.T., 1999. Age-dependent acrylamide neurotoxicity in mice: morphology, physiology, and function. Exp. Neurol. 158, 37-46.

Kupers, R.C., Nuytten, D., Castro-Costa, M., Gybels, J.M., 1992. A time course analysis of the changes in spontaneous and evoked behaviour in a rat model of neuropathic pain. Pain 50, 101-111.

Kupers, R., Yu, W., Persson, J.K., Xu, X.J., Wiesenfeld-Hallin, Z., 1998. Photochemically-induced ischemia of the rat sciatic nerve produces a dose-dependent and highly reproducible mechanical, heat and cold allodynia, and signs of spontaneous pain. Pain 76, 45-59.

Lin, W.M., Hsieh, S.T., Huang, I.T., Griffin, J.W., Chen, W.P., 1997. Ultrastructural localization and regulation of protein gene product 9.5 . NeuroReport 8, 2999-3004.

Lin, Y.W., Tseng, T.J., Lin, W.M., Hsieh, S.T., 2001. Cutaneous nerve terminal degeneration in painful mononeuropathy. Exp. Neurol. 170, $290-296$.

Lindenlaub, T., Sommer, C., 2002. Epidermal innervation density after partial sciatic nerve lesion and pain-related behavior in the rat. Acta. Neuropathol. (Berl.) 104, 137-143.

Ma, W., Bisby, M.A., 2000. Calcitonin gene-related peptide, substance P and protein gene product 9.5 immunoreactive axonal fibers in the rat footpad skin following partial sciatic nerve injuries. J. Neurocytol. 29, $249-262$.

Malmberg, A.B., Basbaum, A.I., 1998. Partial sciatic nerve injury in the mouse as a model of neuropathic pain: behavioral and neuroanatomical correlates. Pain 76, 215-222.

Menovsky, T., van den Bergh Weerman, M., Beek, J.F., 1996. Effect of $\mathrm{CO}_{2}$ milliwatt laser on peripheral nerves: part I. A dose-response study. Microsurgery 17, 562-567.

Menovsky, T., van den Bergh Weerman, M., Beek, J.F., 2000. Effect of $\mathrm{CO}_{2}$ milliwatt laser on peripheral nerves: part II. A histological and functional study. Microsurgery 20, 150-155. 
Morris, S.J., Kunzek, S., Shore, A.C., 1996. The effect of acetylcholine on finger capillary pressure and capillary flow in healthy volunteers J. Physiol. 494, 307-313.

Myers, R.R., James, H.E., Powell, H.C., 1985. Laser injury of peripheral nerve: a model for focal endoneurial damage. J. Neurol. Neurosurg. Psychiatry 48, 1265-1268.

Myers, R.R., Heckman, H.M., Powell, H.C., 1996. Axonal viability and the persistence of thermal hyperalgesia after partial freeze lesions of nerve. J. Neurol. Sci. 139, 28-38.

Nakuda, H., Lynch, C.D.P., McMorran, P.D., 2002. Aggravated reperfusion injury in STZ-diabetic nerve. J. Peripher. Nerv. Syst. 7, 37-43.

Nuytten, D., Kupers, R., Lammens, M., Dom, R., Van Hees, J., Gybels, J., 1992. Further evidence for myelinated as well as unmyelinated fibre damage in a rat model of neuropathic pain. Exp. Brain Res. 91, $73-78$.

Oaklander, A.L., Romans, K., Horasek, S., Stocks, A., Hauer, P., Meyer, R.A., 1998. Unilateral postherpetic neuralgia is associated with bilateral sensory neuron damage. Ann. Neurol. 44, 789-795.

Parry, G.J., Brown, M.J., 1982. Selective fiber vulnerability in acute ischemic neuropathy. Ann. Neurol. 11, 147-154.

Periquet, M.I., Novak, V., Collins, M.P., Nagaraja, H.N., Erdem, S., Nash, S.M., Freimer, M.L., Sahenk, Z., Kissel, J.T., Mendell, J.R., 1999. Painful sensory neuropathy: prospective evaluation using skin biopsy. Neurology 53, 1641-1647.
Rosen, E.D., Raymond, S., Zollman, A., Noria, F., Sandoval-Cooper, M., Shulman, A., Merz, J.L., Castellino, F.J., 2001. Laser-induced noninvasive vascular injury models in mice generate platelet- and coagulation-dependent thrombi. Am. J. Pathol. 158, 1613-1622.

Seltzer, Z., Dubner, R., Shir, Y., 1990. A novel behavioral model of neuropathic pain disorders produced in rats by partial sciatic nerve injury. Pain 43, 205-218.

Vaalasti, A., Tainio, H., Johansson, O., Rechardt, L., 1988. Light and electron microscopic immunocytochemical demonstration of intraepidermal CGRP-containing nerves in human skin. Skin. Pharmacol. $1,225-229$.

Vital, A., Vital, C., Brechenmacher, C., Serise, J.M., Callen, S., Nicolau, H., Videau, J., 1986. Quantitative, histological and ultrastructural studies of peripheral nerve in arteriosclerotic non-diabetic patients. Clin. Neuropathol. 5, 224-229.

Wagner, R., DeLeo, J.A., Heckman, H.M., Myers, R.R., 1995. Peripheral nerve pathology following sciatic cryoneurolysis: relationship to neuropathic behaviors in the rat. Exp. Neurol. 133, 256-264.

Wang, L., Hilliges, M., Jernberg, T., Wiegleb-Edstronom, D., Johansson, O., 1990. Protein gene product 9.5-immunoreactive nerve fibers and cells in human skin. Cell Tissue Res. 261, 25-33.

Woolf, C.J., 2000. Pain. Neurobiol. Dis. 7, 504-510.

Zimmermann, M., 1983. Ethical guidelines for investigations of experimental pain in conscious animals. Pain 16, 109-110. 